\title{
Evaluation of Inferior Vena Cava/Abdominal Aorta Diameter Index in Pulmonary Embolism
}

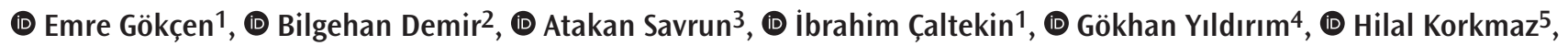 \\ (1) Levent Albayrak ${ }^{1}$, (1) Șeyda Tuba Savrun ${ }^{6}$
}

\author{
1Department of Emergency Medicine, Yozgat Bozok University Faculty of Medicine, Yozgat, Turkey \\ ${ }^{2}$ Clinic of Emergency Medicine, Malatya Research and Training Hospital, Malatya, Turkey \\ ${ }^{3}$ Department of Emergency Medicine, Ordu University Faculty of Medicine, Ordu, Turkey \\ ${ }^{4}$ Clinic of Radiology, Malatya Training and Research Hospital, Malatya, Turkey \\ ${ }^{5}$ Department of Physiology, Gazi University Faculty of Medicine, Ankara, Turkey \\ ${ }^{6}$ Clinic of Emergency Medicine, Ordu University Training and Research Hospital, Ordu, Turkey
}

\begin{abstract}
Aim: Among cardiovascular diseases, pulmonary embolism (PE) is a serious emergency with high mortality. Right ventricular dysfunction due to an excessive increase in pulmonary artery pressure is considered a major cause of death in vigorous PE. We aimed to assess the predictive value of computed tomographic pulmonary angiography (CTPA)-based morphometric measurements of right heart function in the diagnosis of PE.

Materials and Methods: This is a retrospective, case-controlled study. A total of 198 cases were included in the study during the study phase. CTPA results of 102 patients with PE were recorded, and the patients were grouped according to clot localisation. The diameters of the inferior vena cava (IVCA), aorta, pulmonary artery and right and left ventricles were assessed.

Results: IVCA area/aortic area significantly predicted embolism in the main pulmonary artery [area under the curve (AUC) $=0.957, p<0.001]$. The optimal cut-off value was 1.22 with $88 \%$ sensitivity and 90\% specificity. IVCA diameter/aortic diameter significantly predicted embolism in the main pulmonary artery ( $A \cup C=0.955, p<0.001$ ). The optimal cut-off value was 1.1 with $89 \%$ sensitivity and $88 \%$ specificity.

Conclusion: Our study illustrated a remarkable association between the existence and dispersion of PE and morphometric changes in IVCA and aortic ratio parameters measured using CTPA.

Keywords: Pulmonary embolism, inferior vena cava diameter, aortic diameter
\end{abstract}

\section{Introduction}

Pulmonary embolism (PE) is a comparatively widespread emergency among cardiovascular diseases (1). Although PE is a difficult disease to diagnose, rapid diagnosis and appropriate treatment can reduce PE-associated morbidity and mortality (2). Computed tomographic (CT) pulmonary angiography (CTPA) is a diagnostic procedure for suspected cases of PE. CTPA is a method that is non-invasive and has very high sensitivity and specificity (3). Furthermore the presentation of intraluminal clot, CTPA also allows estimation of right ventricular (RV) function in acute PE
$(4,5)$. The main cause of death in PE patients is right ventricular dysfunction (RVD) due to excessive increases in pulmonary artery pressure. The sudden rise in lung vascular resistance leads to RV dilation which changes the contractile properties of the RV myocardium via the Frank-Starling mechanism. RV pressures increase and the patient may present with syncope or hemodynamic deterioration which may result in shock and circulatory arrest due to acute RV insufficiency. Right atrial pressure is a simple and objective index of ventricular function. Inferior vena cava (IVC) diameter is one of the methods used to predict the right atrium pressure $(6,7)$. Therefore, the IVC may

Cite this article as: Gökçen E, Demir B, Savrun A, Çaltekin I, Yıldırım G, Korkmaz H, Albayrak L, Savrun ȘT. Evaluation of Inferior Vena Cava/Abdominal Aorta Diameter Index in Pulmonary Embolism. Eurasian J Emerg Med. 2020;19(2):82-8 
also expand due to increased RV and RA pressures in PE patients. Ordinarily, IVC and descending aortic diameters are similar at the same levels (8). The decrease in left ventricular stroke volume is one of the hemodynamic consequences of PE. The decrease in left ventricular stroke volume will have effects on aortic pressure and size $(9,10)$.

The objectives of this study were to appraise the IVC and aortic dimensions in clinically suspected PE patients, to determine if there is correlation between morphometric measurements of other right ventricular function parameters that can be detected with thoracic CT and to identify the predictive value of these measurements according to clot distribution in PE patients.

\section{Materials and Methods}

This retrospective study was conducted in the departments of emergency medicine between 1/1/2016 and 1/9/2017. This study complied with the declaration of Helsinki and was approved by the local ethics committee (İnönü University) (approval no: 2018/2-21, date: 16.01.2018). CTPA studies and patient files of 129 patients diagnosed with PE radiologically in the emergency department within 21 months were reviewed. Previous clinical features and diagnostic imaging data for each patient were abstracted from electronic patient records. A total of 27 out of 129 patients diagnosed with PE were excluded from the study according to exclusion criteria. CTPA examination was not technically sufficient in 10 of these 27 patients. Five had chronic heart failure, six had cor pulmonale, two had chronic pulmonary thromboembolism, one had malignancy and three had ascending aorta dilatation. Thus, a total of 102 patients formed the PE group. A total of 96 patients with age and gender matching who had no PE or additional pathology in CTPA also formed the control group (Figure 1). The patients included in the study were hemodynamically stable and did not require any vasopressor agent to maintain blood pressures above $100 \mathrm{mmHg}$. The patients included in the study were hemodynamically stable and did not require any vasopressor agent to maintain blood pressure above $100 \mathrm{mmHg}$.

CT scans were evaluated by two radiologists with at least 10 years of experience in thoracic and vascular imaging. PE in the main, right and left pulmonary arteries was defined as group 1. $(n=54)$. PE in the lobar and interlobar arteries was defined as group $2(n=18)$. PE in the segmental and subsegmental branches was defined as group 3 ( $n=30)$. A 128-slice CT scanner (Philips Healthcare, Best, the Netherlands) was used and all patient data were examined on a PACS (picture archiving and communication system) in this study. The cross-sectional areas of the IVC and descending aorta were defined at the plane of the esophageal hiatus. The maximum IVC and aortic diameters were measured on the same slice (Figure 2). The widest wall-to-wall diameters of the right ventricle (RV) and left ventricle (LV) obtained from axial images were also measured. The main pulmonary artery (MPA) wall-to-wall diameter was acquired on axial images at the level of its bifurcation. The right pulmonary artery (RPA) and the left pulmonary artery (LPA) diameters were measured at the largest section after the MPA bifurcation.

Inclusion Criteria: patients older than 18 years with acute PE proven by CTPA.

Exclusion Criteria: definitive diagnosis of cor pulmonale, pulmonary hypertension, pregnancy, malignancy, chronic PE, chronic heart failure, acute coronary syndrome, tricuspid or pulmonary valve pathology, aortopathy or aortic dilation.

\section{Statistical Analysis}

Statistical analysis performed using the SPSS program, version 22.0. The distribution of the variables were evaluated by Kolmogorov-Smirnov and Shapiro-Wilk tests. We used chi-square and t-tests as appropriate to test for baseline characteristics

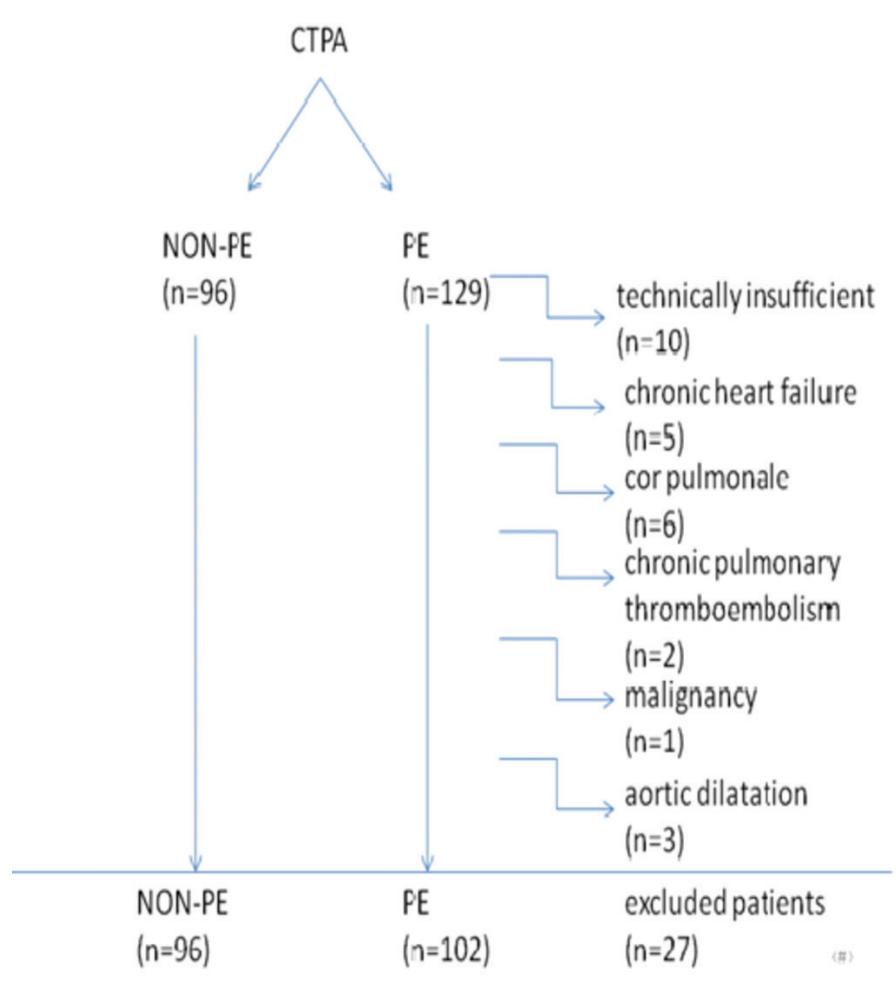

Figure 1. Flowchart shows patient selection

CTPA: Computed tomography pulmonary angiography, PE: Pulmonary Embolism, n: Number 
between the control and study groups. One-way ANOVA test was used to compare multiple groups with normally distributed data. The homogeneity of the variances was evaluated by the Levene test and then post hoc Tukey test was applied. Variables not distributed normally were compared using the Kruskal-Wallis test. ROC analysis was applied to determine the extent to which the tests predicted main pulmonary artery embolization.

\section{Results}

Of the 198 patients included in the study, 96 were in the control group and did not have a PE. The remaining 102 patients were diagnosed with PE. The mean age was $61.3 \pm 16.1$ years in the control group and $62.6 \pm 17.5$ years in the study group $(p>0.05)$. Demographic data of the patients are shown in Table 1. In the

\begin{tabular}{|c|c|c|c|}
\hline & Control & Pulmonary embolism & $p$ \\
\hline \multicolumn{4}{|l|}{ Gender $^{*}$} \\
\hline Male & $42(43.8)$ & $48(47.1)$ & \multirow[t]{2}{*}{0.640} \\
\hline Female & $54(56.3)$ & $54(53)$ & \\
\hline Age $^{*}$ & $61.3 \pm 16.1$ & $62.6 \pm 17.5$ & 0.594 \\
\hline Diabetes Mellitus* & $15(15.6)$ & $20(19.6)$ & 0.463 \\
\hline Hypertension* & $43(44.8)$ & $49(48)$ & 0.647 \\
\hline Dyslipidemia* $^{*}$ & $21(21.9)$ & $27(26.5)$ & 0.451 \\
\hline Ischemic heart disease $^{*}$ & $21(21.9)$ & $26(25.5)$ & 0.550 \\
\hline IVCD (mm) & $23(15-29)$ & $24(17-32)$ & $<0.001$ \\
\hline $\mathrm{AD}(\mathrm{mm})$ & $23(16-28)$ & $21(15-29)$ & $<0.001$ \\
\hline IVCD/AD & $0.96(0.8-1.15)$ & $1.14(0.83-1.61)$ & $<0.001$ \\
\hline IVCA/AA & $0.92(0.64-1.32)$ & $1.3(0.68-2.59)$ & $<0.001$ \\
\hline $\operatorname{MPAD}(\mathrm{mm})$ & $26(16-40)$ & $29.5(22-53)$ & $<0.001$ \\
\hline $\operatorname{RPAD}(\mathrm{mm})$ & $19(13-29)$ & $22(14-36)$ & $<0.001$ \\
\hline LPAD (mm) & $18(13-28)$ & $21(15-36)$ & $<0.001$ \\
\hline RVD (mm) & 37 (26-52) & $43(26-67)$ & $<0.001$ \\
\hline LVD (mm) & $45.5(27-60)$ & $38(23-58)$ & $<0.001$ \\
\hline RVD/LVD & $0.85(0.52-1.41)$ & $1.13(0.71-2.20)$ & $<0.001$ \\
\hline \multicolumn{4}{|c|}{$\begin{array}{l}\text { IVCD: Inferior vena cava diameter, AD: Aorta diameter, IVCA: Inferior vena cava area, AA: Aortic area, MPAD: Mean pulmonary artery diameter, RPAD: Right pulmonary arter } \\
\text { diameter, LPAD: Left pulmonary artery diameter, RVD: Right ventricle diameter, LVD: Left ventricle diameter, CTPA: Computed tomography pulmonary angiogram ekleyelim } \\
\text { Unless otherwise indicated, data are medians, with interquartile ranges in parentheses }\end{array}$} \\
\hline \multicolumn{4}{|c|}{ *Data are given as percentages in parentheses with the number of patients } \\
\hline
\end{tabular}

Table 2. CTPA findings according to clot distribution of pulmonary embolism

\begin{tabular}{|c|c|c|c|c|c|}
\hline & Control & Group 1 & Group 2 & Group 3 & $p$ \\
\hline IVCD (mm) & $22.5 \pm 3.2$ & $25.3 \pm 2.9^{*}$ & $23.3 \pm 2.6$ & $22.9 \pm 3.2^{* * *}$ & $<0.001$ \\
\hline AD (mm) & $23(16-28)$ & $20(15-25)^{*}$ & $22.5(17-25)$ & $23(19-29)^{* *}$ & $<0.001$ \\
\hline IVCD/AD & $0.96(0.8-1.15)$ & $1.22(1.04-1.61)^{*}$ & $1.09(0.92-1.29)^{*}, * *$ & $1.04(0.83-1.14) * *$ & $<0.001$ \\
\hline IVCA/AA & $0.92(0.64-1.32)$ & $1.48(1.09-2.59)^{*}$ & $1.18(0.84-1.67)^{*}, * *$ & $1.08(0.68-1.31)^{* *}$ & $<0.001$ \\
\hline MPAD (mm) & $26(16-40)$ & $31(22-53)^{*}$ & $30(23-36)$ & $29(22-43)$ & $<0.001$ \\
\hline RPAD (mm) & $19(13-29)$ & $23(14-32)^{*}$ & $21.5(15-33)$ & $21.5(14-36)$ & $<0.001$ \\
\hline LPAD (mm) & $18(13-28)$ & $22(16-27)^{*}$ & $21(16-26)$ & $20(15-36)^{* * *}$ & $<0.001$ \\
\hline RVD (mm) & $37(26-52)$ & $45.5(34-58) *$ & $41(32-56)$ & $40.5(26-67)^{* *}$ & $<0.001$ \\
\hline LVD (mm) & $44.7 \pm 6.7$ & $36.4 \pm 6.4^{*}$ & $39.8 \pm 6.7^{*}$ & $41.1 \pm 8.4^{* *}$ & $<0.001$ \\
\hline RVD/LVD & $0.85(0.52-1.41)$ & $1.26(0.78-2.20)^{*}$ & $1.05(0.73-1.83)^{*}$ & $0.99(0.71-1.80)^{*}, * * *$ & $<0.001$ \\
\hline
\end{tabular}

IVCD: Inferior vena cava diameter, AD: Aorta diameter, IVCA: Inferior vena cava area, AA:Aortic area, MPAD: Main pulmonary artery diameter, RPAD: Right pulmonary artery diameter, LPAD: Left pulmonary artery diameter, RVD: Right ventricle diameter, LVD: Left ventricle diameter, CTPA: Computed tomography pulmonary angiogram

${ }^{*} \mathrm{p}<0.05$ for groups compared with the control group, ** $p<0.05$ for groups compared with the group 1 
study group, 54 patients had embolism in the main pulmonary artery, 18 patients had lobar and interlobar PE and 30 patients had segmental or subsegmental emboli.

The IVC diameter (IVCD)/Aortic diameter (AD) ratio was 1.14 (0.83-1.61) and 0.96 (0.8-1.15) in the study and control groups, respectively. The IVC area (IVCA)/Aortic Area (AA) ratio was 1.3 (0.68-2.59) and 0.92 (0.64-1.32) in the study and control groups, respectively (Table 1$)$. When the IVCD/AD ratio and IVCA/AA ratio were examined according to clot distribution, the highest rates were observed in group 1 [1.04 (0.83-1.14), 1.08 (0.68-1.31) respectively]. IVCD/AD and IVCA/ AA ratios were significantly higher in patients with central PE $(p<0.001)$ (Table 2). IVCD was measured as 24 (17-32) $\mathrm{mm}$ and 23 (15-29) $\mathrm{mm}$ in the study and control groups, respectively. AD was 21 (15-29) mm and 23 (1628) $\mathrm{mm}$ in the study and control groups, respectively (Table 1).

MPA diameter (MPAD) was 29.5 (22-53) mm and 26 (16-40) mm in the study and control groups, respectively. RPA diameter (RPAD) was found to be 22 (14-36) $\mathrm{mm}$ and 19 (13-29) $\mathrm{mm}$ in the study and control groups, respectively. LPA diameter (LPAD) was found to be 21 (15-36) $\mathrm{mm}$ and 18 (13-28) $\mathrm{mm}$ in the study and control groups, respectively (Table 1). When MPAD, RPAD and LPAD were examined according to clot distribution, the highest values were observed in group 1 [31 (22-53), 23 (14-32), 22 (16-27) respectively]. MPAD, RPAD and $L P A D$ were significantly higher in patients with central PE $(p<0.001)$ (Table 2).

RV diameter was found to be 29.5 (22-53) mm and 26 (16-40) $\mathrm{mm}$ in the study and control groups, respectively. LV diameter was found to be 38 (23-58) $\mathrm{mm}$ and 45.5 (27-60) $\mathrm{mm}$ in the study and control groups, respectively (Table 1). When RV diameter measurement values were examined according to clot distribution, the highest values were observed in group 1 [45.5 (34-58)]. When LV diameter measurement values were examined according to clot distribution, the lowest values were

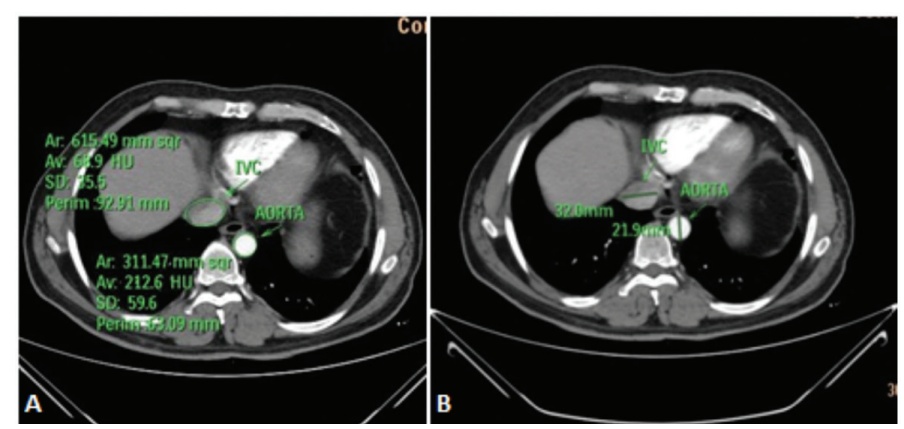

Figure 2. (A): The cross-sectional areas of the suprahepatic IVC and descending aorta were measured on a uniaxial image at the level of the esophageal hiatus. (B): The maximum IVC and aortic diameters were measured on the same slice.

IVC: Inferior vena cava observed in group 1 [36.4 \pm 6.4$]$. While RV diameter was found to be significantly higher in patients with central PE, LV diameter was significantly lower $(p<0.001, p<0.001$ respectively) (Table 2). RV diameter/LV diameter (RV/LV ratio) was found to be 1.14 (0.83-1.61) and 0.96 (0.8-1.15) in the study and control groups, respectively [1.13 (0.71-2.20), 0.85 (0.52-1.41) respectively] (Table 1). RV/LV ratio was significantly higher in patients with central PE $(p<0.001)$ (Table 2).

\section{Roc Analysis}

IVCA/AA significantly predicted embolism in the main pulmonary artery (AUC $=0.957, p<0.001$ ). The optimal cut-off value was calculated to be 1.22 with $88 \%$ sensitivity and $90 \%$ specificity (Figure 3).

IVCD/AD significantly predicted embolism in the main pulmonary artery (AUC $=0.955, p<0.001$ ). The optimal cut-off value was calculated as 1.1 with $89 \%$ sensitivity and $88 \%$ specificity (Figure 3).

RVD/LVD significantly predicted embolism in the main pulmonary artery $(A \cup C=0.874, p<0.001)$. The optimal cut-off value was calculated to be 1.1 with $79 \%$ sensitivity and $79 \%$ specificity (Figure 3).

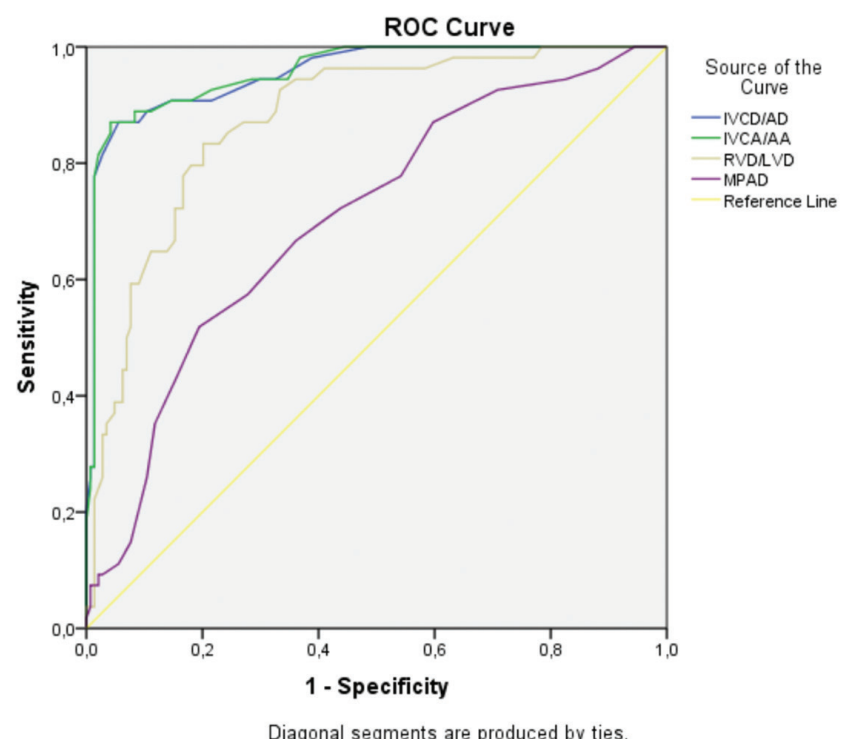

Figure 3. ROC analysis: The predicted value of IVCD/AD, IVCD/ $A A, R V D / L V D$ and MPAD in specifying the main pulmonary artery embolism. (IVCD/AD: AUC $=0.955, p<0.001$, IVCA/AA: $A U C=0.957$, $p<0.001, \quad R V D / L V D: \quad A U C=0.874, p<0.001, \quad M P A D: \quad A U C=0.705$, $\mathrm{p}<0.001)$

ROC: Receiver operating characteristic, IVCD/AD: Inferior vena cava diameter/aortic diameter, IVCA/AA: Inferior vena cava diameter/ aortic diameter, RVD/LVD: Right ventricle diameter/left ventricle diameter, MPAD: Main pulmonary artery diameter, AUC: Area under curve 
MPAD significantly predicted embolism in the main pulmonary artery $(A \cup C=0.705, p<0.001)$. The optimal cut-off value was calculated as 28.5 with $67 \%$ sensitivity and 64\% specificity (Figure 3).

\section{Discussion}

Acute PE is an important emergency with high morbidity and mortality among cardiovascular diseases despite many medical developments. The clinician should always be alert to the clinically suspected PE because early diagnosis and urgent treatment can reduce PE related morbidity and mortality (11).

In this study, we investigated the relationship between pulmonary artery clot distribution and IVC/aortic ratio parameters in patients with PE. These include the IVC/AD ratio and the ratio of IVC to aortic cross-sectional area (IVCA/AA). In our study, the highest values of these parameters were seen in the main pulmonary artery group. The lobar group was in the second rank and the segmental or subsegmental group was observed to be in the third rank. A statistically significant difference was found when groups 1 and 2 were compared with the control group in terms of IVCA/AA and IVCD/AD ratios. In addition, there was a statistically remarkable discrepancy among groups 2 and 3 and group 1 in terms of IVCA/AA and IVC/AD ratios. These results emphasize that the values of these parameters will increase if the PE is localized more centrally. Among the evaluated parameters, there was a higher sensitivity and specificity in IVCA/AA and IVCD/AD. IVCA/ AA ratio cut-off of 1.22 ( $A \cup C=0.957$ ) and an IVCD/AD ratio cutoff of 1.1 ( $A \cup C=0.955$ ) were calculated for detection of main pulmonary artery embolism. These findings suggest that these parameters have a high correlation with the pulmonary artery occlusion grade.

The main consequences of pulmonary thromboembolism are hemodynamic (10). Left ventricular stroke volume is reduced as a result of the shift of the interventricular septum to the left, impaired left ventricular filling pressures and the diastolic left ventricular dysfunction. Sympathetic activation and systemic vasoconstriction develop as a compensatory mechanism $(10,12)$. Possibly all these events may lead to a modest decrease in aortic size. In fact, a decrease in the aortic diameter was observed in the first group. Once the clot flows to the pulmonary artery, RV and RA pressures increase; this is transmitted to the IVC and results in IVC dilatation $(9,13)$. In this research, a statistically prominent change was detected in the first group compared to the control group in terms of aorta diameter (AD) and inferior vena cava diameter (IVCD). Breathing maneuvers are important in IVC measurements. Normally, in the inspiratory phase of the respiratory cycle, venous return increases towards the right heart due to negative intrapleural pressure. This leads to an increase in
IVC diameter. An expanded IVC with a lack of collapse by $>50 \%$ in the inspiratory phase suggests elevated RA pressure. The thoracic $\mathrm{CT}$ is performed at the end of the inspiratory phase and therefore better represents the measurements $(6,14)$.

PE increases pulmonary vascular resistance and may lead to RVD. International ECHO multicenter studies have shown that in PE patients, RVD is a major determinant of short-term mortality $(15,16)$. Follow-up of right ventricular functions plays an important role in the treatment of PE. ESC recommends transthoracic echocardiogram (TTE) in hemodynamically unstable patients for the diagnosis of PE (17). In addition, TTE can be used in risk classification for evaluation of right ventricular functions in the differentiation of submassive and low-risk PE in hemodynamically stable patients $(17,18)$. Previous studies have also demonstrated the ability of PCTA findings to identify RVD in PE $(19,20)$. The RVD/LVD ratio is one of the markers of RVD measured by CTPA $(21,22)$. In our study, we found the sensitivity and specificity of the IVC/aortic ratio parameters in detecting the main PE were higher than the sensitivity and specificity of the RVD/LVD ratio. Additionally, we demonstrated that the IVCD/ $A D$ and IVCA/AA ratio increased when PE was located more centrally. The central localization of the clot in the pulmonary artery is directly proportional to the load on the right ventricle $(10,21)$. Therefore, an increase in IVCD/AD and IVCA/AA ratio may be indicative of RVD. In this context, we believe that these parameters are useful and easily accessible markers that can be detected by CTPA in determining RVD in patients with PE.

Earlier studies have suggested that an RVD/LVD ratio $>1.0$ was an independent risk factor for RVD $(22,23)$. It can be considered as a reliable indicator in the evaluation of 30-day mortality in patients with acute PE (24). In our study, an RVD/LVD ratio cut-off of 1.1 was calculated for the diagnosis of main pulmonary artery embolism. We found the highest values of RVD/LVD ratio in group 1. There was no remarkable statistical difference among groups 1 and 2 from the standpoint of RVD/LVD ratio, but there was a statistically remarkable difference among group 1 and group 3. Furthermore, there was a statistically remarkable difference in both group 1 and group 2 compared to the control group in terms of RVD/LVD ratios. This obtained data propose that an increase in RVD/LVD ratio can be expected in more centrally located PE cases.

MPAD was higher in group 1 patients. When MPAD, RPAD and LPAD were analysed according to clot distribution in PE patients, significant statistical change was detected between group 1 and the control group. In a previous study, the MPAD threshold value for patients with cardiopulmonary disease was calculated to be $28.6 \mathrm{~mm}$ with a mean pulmonary artery pressure of $>18 \mathrm{~mm} \mathrm{Hg}$ (25). In another study, the MPAD threshold value for patients with 
parenchymal lung disease was calculated to be $>29.0 \mathrm{~mm}$ with a sensitivity of $84 \%$ and a specificity of $89 \%$ in predicting a mean pulmonary artery pressure of $>20 \mathrm{~mm} \mathrm{Hg}$ (26). In our study, the MPAD cut-off of $28.5 \mathrm{~mm}$ was calculated for the diagnosis of main pulmonary artery embolism. The MPAD significantly predicted embolism in the main pulmonary artery.

\section{Study Limitations}

Our study had some limitations. First of all, our study was retrospectively reviewed. Data on follow-up and outcome of patients could not be included in the study. In our study, a consistent gold standard method of RVD was not determined. Thus, similar to previous studies, we assumed that patients with central embolism had a higher incidence of RVD than patients with peripheral embolism and those without PE (2). In the literature, there are many studies indicating the importance of echocardiography in the evaluation of right ventricular functions and mortality risk classification in PE patients $(27,28)$. In our study, echocardiographic data could not be used to evaluate right ventricular functions. However, future studies should compare echocardiographic data with IVC/aortic ratio parameters in PE patients.

\section{Conclusion}

The results of the study imply that the IVC/aortic ratio parameters predict more centrally located PEs that will cause right heart tension. We believe that IVC/aortic ratio parameters reflecting hemodynamic changes in response to the severity of pulmonary artery occlusion in PE patients may contribute to a more comprehensive risk classification in these patients. Prognostic values of these parameters should be determined prospectively in future studies. Furthermore, the measurement of the MPAD and RVD/LVD ratio using CTPA is a valuable adjunct for the prediction of RVD and the evaluation of the severity of acute PE.

\section{Ethics}

Ethics Committee Approval: This study complied with the declaration of Helsinki and was approved by the local ethics committee (İnönü University) (approval no: 2018/2-21, date: 16.01.2018).

Informed Consent: Retrospective study.

Peer-review: Internally and externally peer-reviewed.

\section{Authorship Contributions}

Surgical and Medical Practices: E.G., A.S., G.Y., Concept: E.G., Design: E.G., I.C.., Data Collection or Processing: E.G., B.D., I.Ç., G.Y., Analysis or Interpretation: E.G., B.D., A.S., I.C.., G.Y., H.K.,
L.A., Ş.T.S., Literature Search: E.G., B.D., A.S., I.Ç., H.K., L.A., Ş.T.S., Writing: E.G.

Conflict of Interest: No conflict of interest was declared by the authors.

Financial Disclosure: The authors declared that this study received no financial support.

\section{References}

1. Köse N, Yıldııım T, Akın F, Yıldııım SE, Altun I. Prognostic role of NLR, PLR, and LMR in patients with pulmonary embolism. Bosn J Basic Med Sci. 2019;20:248-53.

2. Henzler T, Krissak R, Reichert M, Sueselbeck T, Schoenberg SO, Fink C. Volumetric analysis of pulmonary CTA for the assessment of right ventricular dysfunction in patients with acute pulmonary embolism. Academic Radiol. 2010;17:309-15.

3. Schissler AJ, Rozenshtein A, Kulon ME, Pearson GD, Green RA, Stetson PD, et al. CT pulmonary angiography: increasingly diagnosing less severe pulmonary emboli. PLoS One. 2013;8:e65669.

4. Trujillo-Santos J, den Exter PL, Gómez V, Del Castillo H, Moreno C, van der Hulle T, et al. Computed tomography-assessed right ventricular dysfunction and risk stratification of patients with acute non-massive pulmonary embolism: systematic review and meta-analysis. J Thromb Haemost. 2013;11:1823-32.

5. Lyhne MD, Schultz JG, MacMahon PJ, Haddad F, Kalra M, Tso DM, et al. Septal bowing and pulmonary artery diameter on computed tomography pulmonary angiography are associated with short-term outcomes in patients with acute pulmonary embolism. Emerg Radiol. 2019;26:623-30.

6. Rudski LG, Lai WW, Afilalo J, Hua L, Handschumacher MD, Chandrasekaran $\mathrm{K}$, et al. Guidelines for the echocardiographic assessment of the right heart in adults: a report from the American Society of Echocardiography: endorsed by the European Association of Echocardiography, a registered branch of the European Society of Cardiology, and the Canadian Society of Echocardiography. J Am Soc Echocardiogr. 2010;23:685-713.

7. Pinsky MR. The right ventricle: interaction with the pulmonary circulation. Crit Care. 2016;20:266.

8. Giorgi B, Mollet NR, Dymarkowski S, Rademakers FE, Bogaert J. Clinically suspected constrictive pericarditis: MR imaging assessment of ventricular septal motion and configuration in patients and healthy subjects. Radiology. 2003;228:417-24.

9. D’Alto M, Scognamiglio G, Dimopoulos K, Bossone E, Vizza D, Romeo E, et al. Right heart and pulmonary vessels structure and function. Echocardiography. 2015;32:(Suppl 1):3-10.

10. Wood KE. Major pulmonary embolism: review of a pathophysiologic approach to the golden hour of hemodynamically significant pulmonary embolism. Chest. 2002;121:877-905.

11. Konstantinides SV, Barco S, Lankeit M, Meyer G. Management of pulmonary embolism: an update. J Am Coll Cardiol. 2016;67:976-90.

12. Tapson VF. Advances in the diagnosis and treatment of acute pulmonary embolism. F1000 Med Rep. 2012;4:9.

13. Brennan JM, Blair JE, Goonewardena S, Ronan A, Shah D, Vasaiwala S, et al. Reappraisal of the use of inferior vena cava for estimating right atrial pressure. J Am Soc Echocardiogr. 2007;20:857-61.

14. Kircher BJ, Himelman RB, Schiller NB. Noninvasive estimation of right atrial pressure from the inspiratory collapse of the inferior vena cava. Am J Cardiol. 1990;66:493-6.

15. Khemasuwan D, Yingchoncharoen T, Tunsupon P, Kusunose K, Moghekar A, Klein A, et al. Right ventricular echocardiographic parameters are associated with mortality after acute pulmonary embolism. J Am Soc Echocardiogr. 2015;28:355-62. 
16. Barrios D, Morillo R, Lobo JL, Nieto R, Jaureguizar A, Portillo AK, et al. Assessment of right ventricular function in acute pulmonary embolism. Am Heart J. 2017;185:123-9.

17. Dutta T, Frishman WH, Aronow WS. Echocardiography in the evaluation of pulmonary embolism. Cardiol Rev. 2017;25:309-14.

18. Saric M, Armour AC, Arnaout MS, Chaudhry FA, Grimm RA, Kronzon I, et al. Guidelines for the use of echocardiography in the evaluation of a cardiac source of embolism. J Am Soc Echocardiogr. 2016;29:1-42.

19. Beenen LFM, Bossuyt PMM, Stoker J, Middeldorp S. Prognostic value of cardiovascular parameters in computed tomography pulmonary angiography in patients with acute pulmonary embolism. Eur Respir J. 2018;52.

20. Doğan H, de Roos A, Geleijins J, Huisman MV, Kroft LJ. The role of computed tomography in the diagnosis of acute and chronic pulmonary embolism. Diagn Interv Radiol. 2015;21:307-16.

21. Huang J, Gang W. Evaluation of Multiple-slice CT Pulmonary Angiography on Right Ventricular Parameters in Acute Pulmonary Embolism. Chinese Journal of Medical Imaging. 2017;25:270-3.

22. Jia D, Zhou XM, Hou G. Estimation of right ventricular dysfunction by computed tomography pulmonary angiography: a valuable adjunct for evaluating the severity of acute pulmonary embolism. J Thromb Thrombolysis. 2017;43:271-8.

23. Findik S, Erkan L, Light RW, Uzun O, Atici AG, Akan H. Massive pulmonary emboli and CT pulmonary angiography. Respiration. 2008;76:403-12.

24. Bach AG, Nansalmaa B, Kranz J, Taute BM, Wienke A, Schramm D, et al. CT pulmonary angiography findings that predict 30-day mortality in patients with acute pulmonary embolism. Eur J Radiol. 2015;84:332-7.

25. Kuriyama K, Gamsu G, Stern RG, Cann CE, Herfkens RJ, Brundage BH. CT-determined pulmonary artery diameters in predicting pulmonary hypertension. Invest Radiol. 1984;19:16-22.

26. Tan RT, Kuzo R, Goodman LR, Siegel R, Haasler GR, Presberg KW. Utility of CT scan evaluation for predicting pulmonary hypertension in patients with parenchymal lung disease. Chest. 1998;113:1250-6.

27. Bikdeli B, Lobo JL, Jiménez D, Green P, Fernández-Capitán C, BuraRiviere A, et al. Early Use of Echocardiography in Patients With Acute Pulmonary Embolism: Findings From the RIETE Registry. J Am Heart Assoc. 2018;7:e009042.

28. Cohen DM, Winter M, Lindenauer PK, Walkey AJ. Echocardiogram in the evaluation of hemodynamically stable acute pulmonary embolism: national practices and clinical outcomes. Ann Am Thorac Soc. 2018;15:581-8. 